Proyecciones Journal of Mathematics Vol. 31, No 1, pp. 29-38, March 2012. Universidad Católica del Norte Antofagasta - Chile

\title{
Improving some sequences convergent to Euler-Mascheroni constant
}

\author{
NECDET BATIR \\ Nevşehır University, Nevşehır, TURKEY \\ and \\ CHAO-PING CHEN \\ Henan Polytechnic University, CHINA \\ Received : November 2011. Accepted : December 2011
}

\begin{abstract}
We obtain the following very fast sequences convergent to EulerMascheroni constant:

$\theta_{n}=H_{n}-\log \left(n+\frac{1}{2}+\frac{1}{24 n}-\frac{1}{48 n^{2}}+\frac{23}{5760 n^{3}}+\frac{17}{3840 n^{4}}-\frac{10099}{2903040 n^{5}}\right)$

and

$\phi_{n}=H_{n}-\log \left(n+\frac{1}{2}+\frac{1}{24\left(n+\frac{1}{2}\right)}-\frac{37}{5760\left(n+\frac{1}{2}\right)^{3}}+\frac{10313}{2903040\left(n+\frac{1}{2}\right)^{5}}\right)$,

where $H_{n}$ are the harmonic numbers defined by $H_{n}=1+\frac{1}{2}+\frac{1}{3}+\ldots+\frac{1}{n}$.

subjclass [2000] : Primary 11Y60; Secondary 40A05.
\end{abstract}

Keywords : Euler-Mascheroni constant, harmonic numbers, inequalities, asymptotic expansion. 


\section{Introduction}

Euler's constant ( or Euler Mascheroni constant) $\gamma$ was introduced by Leonhard Euler (1707-1783) in 1734 as the limit of the sequence

$$
D_{n}=1+\frac{1}{2}+\frac{1}{3}+\ldots+\frac{1}{n}-\log n .
$$

This constant is known to be the third most important mathematical constant, next to $\pi$ and $e$. It appears in a lot of places in mathematics such as number theory, analysis, theory of probability, special functions, and differential equations. The convergence of $D_{n}$ to $\gamma$ is very slowly since

$$
\frac{1}{2(n+1)}<D_{n}-\gamma<\frac{1}{2 n} \quad \text { (R.M. Young [12]), }
$$

which shows that it converges to $\gamma$ as $n^{-1}$. A faster convergent sequence to $\gamma$ were introduced by DeTemle in $[10,11]$. He proved that the sequence $R_{n}$ defined by

$$
R_{n}=1+\frac{1}{2}+\frac{1}{3}+\ldots+\frac{1}{n}-\log \left(n+\frac{1}{2}\right) .
$$

converges to $\gamma$ with the speed like $n^{-2}$, since

$$
\frac{1}{24(n+1)^{2}}<R_{n}-\gamma<\frac{1}{24 n^{2}}
$$

Recently Chen [3] obtained sharp form of (1.3) as follows: For all $n \in \mathbf{N}$

$$
\frac{1}{24(n+a)^{2}}<R_{n}-\gamma<\frac{1}{24(n+b)^{2}}
$$

with the best possible constants

$$
a=\frac{1}{\sqrt{24(1-\gamma-\log (3 / 2))}}=0.55106 \ldots, \text { and } b=\frac{1}{2} .
$$

In 1997, Negoi [9] introduced the sequence

$$
T_{n}=1+\frac{1}{2}+\frac{1}{3}+\ldots+\frac{1}{n}-\log \left(n+\frac{1}{2}+\frac{1}{24 n}\right)
$$

and showed that

$$
\frac{1}{48(n+1)^{3}}<T_{n}-\gamma<\frac{1}{48 n^{3}}
$$


which shows that the approximation $T_{n} \approx \gamma$ has a significient superiority over the approximation $R_{n} \approx \gamma$. For other faster convergences of EulerMascheroni constant we refer to $[1,3,5,6,7,8]$. To accelerate the sequence $\left(T_{n}\right)$, Chen and Mortici [2] established the following approximation formula

$$
\gamma \approx H_{n}-\log \left(n+\frac{1}{2}+\frac{1}{24 n}-\frac{1}{48 n^{2}}+\frac{23}{5760 n^{3}}+\ldots\right)
$$

Our first aim here is to improve (1.4) and obtain bounds for $H_{n}$ in this form. Our second aim is to establish similar formulas to improve $\left(R_{n}\right)$. For this purpose we shall consider the following sequences:

$$
A_{n}=H_{n}-\log \left(n+\frac{1}{2}+\frac{1}{24 n}+\frac{\alpha}{n^{2}}+\frac{\beta}{n^{3}}+\frac{\delta}{n^{4}}+\frac{\epsilon}{n^{5}}\right),
$$

and

$$
\mathrm{B}_{n}=H_{n}-\log \left(n+\frac{1}{2}+\frac{a}{n+\frac{1}{2}}+\frac{b}{\left(n+\frac{1}{2}\right)^{2}}+\frac{c}{\left(n+\frac{1}{2}\right)^{3}}+\frac{c}{\left(n+\frac{1}{2}\right)^{3}}+\frac{d}{\left(n+\frac{1}{2}\right)^{4}}+\frac{e}{\left(n+\frac{1}{2}\right)^{5}}\right),
$$

where $\alpha, \beta, \delta, \epsilon$ and $a, b, c, d, e$ are real parameters. Precisely, we introduce the sequences $\left(\theta_{n}\right)$ and $\left(\phi_{n}\right)$ by

$\theta_{n}=H_{n}-\log \left(n+\frac{1}{2}+\frac{1}{24 n}-\frac{1}{48 n^{2}}+\frac{23}{5760 n^{3}}+\frac{17}{3840 n^{4}}-\frac{10099}{2903040 n^{5}}\right)$,

and

$\phi_{n}=H_{n}-\log \left(n+\frac{1}{2}+\frac{1}{24\left(n+\frac{1}{2}\right)}-\frac{37}{5760\left(n+\frac{1}{2}\right)^{3}}+\frac{10313}{2903040\left(n+\frac{1}{2}\right)^{5}}\right)$,

both of which converge to $\gamma$ like $n^{-7}$, since

$$
\lim _{n \rightarrow \infty} n^{7}\left(\theta_{n}-\gamma\right)=-\frac{2501}{1161216}
$$

and

$$
\lim _{n \rightarrow \infty} n^{7} \phi_{n}=-\frac{5509121}{1393459200} .
$$

In order to prove our main results we need the following lemma, which is a strong tool to measure and improve the speed of convergence of some sequences having limit equal to zero. 
Lemma 1.1. If $\left(s_{n}\right)$ is convergent to zero and there exists the limit

$$
\lim _{n \rightarrow \infty} n^{k}\left(s_{n}-s_{n+1}\right)=c \in \mathbf{R}
$$

then there exists the limit

$$
\lim _{n \rightarrow \infty} n^{k-1} s_{n}=\frac{c}{k-1}
$$

see [4]. From this lemma it is clear that the speed of convergence of the sequence $\left(s_{n}\right)$ is as higher as the value of $k$ satisfying (1.6) is as greater as.

\section{Main results}

Let $A_{n}$ be the sequence defined by (1.5). We are interested to find the values of $\alpha, \beta, \delta$ and $\epsilon$ which provide the fastest sequence $A_{n}$. First, let us write

$$
\begin{aligned}
& \mathrm{A}_{n}-A_{n+1}=-\frac{1}{n+1}-\log \left(n+\frac{1}{2}+\frac{1}{24 n}+\frac{\alpha}{n^{2}}+\frac{\beta}{n^{3}} \frac{\delta}{n^{4}}+\frac{\epsilon}{n^{5}}\right) \\
& +\log \left(n+1+\frac{1}{2}+\frac{1}{24(n+1)}+\frac{\alpha}{(n+1)^{2}}+\frac{\delta}{(n+1)^{4}}+\frac{\epsilon}{(n+1)^{5}}\right) .
\end{aligned}
$$

We are concentrated to compute a limit of the form (1.6). In order to do this we use a computer software to obtain the following power series representation in $\frac{1}{n}$ :

$$
\begin{gathered}
\mathrm{A}_{n}-A_{n+1}=\left(-\frac{1}{16}-3 \alpha\right) \frac{1}{n^{4}}+\left(\frac{263}{1440}+8 \alpha-4 \beta\right) \frac{1}{n^{5}} \\
+\left(-\frac{139}{384}-\frac{385 \alpha}{24}+\frac{25 \beta}{2}-5 \delta\right) \frac{1}{n^{6}}+\left(\frac{3685}{6048}+\frac{229 \alpha}{8}+3 \alpha^{2}-\frac{115 \beta}{4}+18 \delta-6 \epsilon\right) \frac{1}{n^{7}} \\
+\left(-\frac{8663}{9216}-\frac{27517 \alpha}{576}-14 \alpha^{2}+\frac{1379 \beta}{24}+7 \alpha \beta-\frac{1127 \delta}{24}+\frac{49 \epsilon}{2}\right) \frac{1}{n^{8}}+O\left(n^{-9}\right) .
\end{gathered}
$$

By Lemma 1.1 faster convergences are obtained by imposing the conditions that the first four coefficients vanish. Now this results in 


$$
\left\{\begin{array}{l}
-\frac{1}{16}-3 \alpha=0 \\
\frac{263}{1440}+8 \alpha-4 \beta=0 \\
\frac{139}{384}-\frac{385 \alpha}{24}+\frac{25 \beta}{2}-5 \delta=0 \\
\frac{3685}{6048}+\frac{229 \alpha}{8}+3 \alpha^{2}-\frac{115 \beta}{4}+18 \delta-6 \epsilon=0 \\
-\frac{8663}{9216}-\frac{27517 \alpha}{576}-14 \alpha^{2}+\frac{1379 \beta}{24}+7 \alpha \beta-\frac{1127 \delta}{24}+\frac{49 \epsilon}{2}=0
\end{array}\right.
$$

namely,

$$
\alpha=-\frac{1}{48}, \quad \beta=\frac{23}{5760}, \delta=\frac{17}{3840}, \text { and } \epsilon=-\frac{10099}{2903040} .
$$

These solutions correspond to the following sequence

$\theta_{n}=H_{n}-\log \left(n+\frac{1}{2}+\frac{1}{24 n}-\frac{1}{48 n^{2}}+\frac{23}{5760 n^{3}}+\frac{17}{3840 n^{4}}-\frac{10099}{2903040 n^{5}}\right)$.

By replacing the solutions (2.1) above

$$
\theta_{n}-\theta_{n+1}=-\frac{2501}{165888 n^{8}}+O\left(n^{-9}\right)
$$

Now we can state the following

Theorem 2.1. Let $\left(\theta_{n}\right)$ be the sequence defined by (2.2). Then

$$
\lim _{n \rightarrow \infty} n^{8}\left(\theta_{n}-\theta_{n+1}\right)=-\frac{2501}{165888} \text { and } \lim _{n \rightarrow \infty} n^{7}\left(\theta_{n}-\gamma\right)=-\frac{2501}{1161206},
$$

namely, the speed of convergences of the sequence $\left(\theta_{n}\right)$ is like $n^{-7}$

Let $\left(B_{n}\right)$ be as defined by (1). Then we have

$$
\begin{aligned}
& B_{n}-B_{n+1}=-\frac{1}{n+1}-\log \left(n+\frac{1}{2}+\frac{a}{\left(n+\frac{1}{2}\right)}+\frac{b}{\left(n+\frac{1}{2}\right)^{2}}+\frac{c}{\left(n+\frac{1}{2}\right)^{3}}+\frac{d}{\left(n+\frac{1}{2}\right)^{4}}\right. \\
& \left.+\frac{e}{\left(n+\frac{1}{2}\right)^{5}}\right)+\log \left((n+1)+\frac{1}{2}+\frac{a}{\left(n+\frac{3}{2}\right)}+\frac{b}{\left(n+\frac{3}{2}\right)^{2}}+\frac{c}{\left(n+\frac{3}{2}\right)^{3}}+\frac{d}{\left(n+\frac{3}{2}\right)^{4}}+\frac{e}{\left(n+\frac{3}{2}\right)^{5}}\right) .
\end{aligned}
$$


Using again a computer software we get

$$
\begin{aligned}
& B_{n}-B_{n+1}=\left(\frac{1}{12}-2 a\right) \frac{1}{n^{3}}+\left(-\frac{1}{4}+6 a-3 b\right) \frac{1}{n^{4}} \\
& +\left(\frac{41}{80}-13 a+2 a^{2}+12 b-4 c\right) \frac{1}{n^{5}} \\
& +\left(-\frac{43}{48}+25 a-10 a^{2}-\frac{65 b}{2}+5 a b+20 c-5 d\right) \frac{1}{n^{6}} \\
& +\left(\frac{645}{448}-\frac{363 a}{8}+\frac{65 a^{2}}{2}-2 a^{3}+75 b-30 a b+3 b^{2}-65 c+6 a c+30 d-6 e\right) \frac{1}{n^{7}} \\
& \left(-\frac{141}{64}+\frac{637 a}{8}-\frac{175 a^{2}}{2}+14 a^{3}-\frac{2541 b}{16}+\frac{455 a b}{4}-7 a^{2} b-21 b^{2}+175 c\right. \\
& \left.-42 a c+7 b c-\frac{455 d}{4}+7 a d+42 e\right) \frac{1}{n^{8}}+O\left(n^{-9}\right) .
\end{aligned}
$$

According to Lemma 1.1 we can see that the fastest sequence $\phi_{n}$ is obtained in the case when as many of the first coefficients of (2.3) is cancelled. As we have five paremeters $a, b, c, d, e$, they produce the best result if and only if

$$
\begin{aligned}
& \frac{1}{12}-2 a=0, \\
& -\frac{1}{4}+6 a-3 b=0, \\
& \frac{41}{80}-13 a+2 a^{2}+12 b-4 c=0, \\
& -\frac{43}{48}+25 a-10 a^{2}-\frac{65 b}{2}+5 a b+20 c-5 d=0, \\
& \frac{645}{448}-\frac{363 a}{8}+\frac{65 a^{2}}{2}-2 a^{3}+75 b-30 a b+3 b^{2}-65 c \\
& +6 a c+30 d-6 e=0, \\
& -\frac{141}{64}+\frac{637 a}{8}-\frac{175 a^{2}}{2}+14 a^{3}-\frac{2541 b}{16}+\frac{455 a b}{4}-7 a^{2} b \\
& -21 b^{2}+175 c-42 a c+7 b c-\frac{455 d}{4}+7 a d+42 e=0 .
\end{aligned}
$$


From these we obtain the following solutions:

$$
a=\frac{1}{24}, \quad b=0, \quad c=-\frac{37}{5760}, \quad d=0, \quad e=\frac{10313}{2903040},
$$

and these solutions correspond to the following sequence

$$
\phi_{n}=H_{n}-\log \left(n+\frac{1}{2}+\frac{1}{24\left(n+\frac{1}{2}\right)}-\frac{37}{5760\left(n+\frac{1}{2}\right)^{3}}+\frac{10313}{2903040\left(n+\frac{1}{2}\right)^{5}}\right) .
$$

By replacing the solutions given in (2.5) in (2.4) we get

$$
\phi_{n}-\phi_{n+1}=-\frac{5509121}{174182400 n^{8}}+O\left(n^{-9}\right) .
$$

These can be summarized as follow.

Theorem 2.2. Let $\left(\phi_{n}\right)$ be the sequence given by (2.6). Then it holds that

$$
\lim _{n \rightarrow \infty} n^{8}\left(\phi_{n}-\phi_{n+1}\right)=-\frac{5509121}{174182400} \text { and } \lim _{n \rightarrow \infty} n^{7} \phi_{n}=-\frac{5509121}{1393459200}
$$

that is, the speed of convergence of $\left(\phi_{n}\right)$ is like $n^{-7}$.

Theorem 2.3. Let the sequences $\left(\theta_{n}\right)$ and $\left(\phi_{n}\right)$ be as defined (2.2) and (2.6). Then, both $\left(\theta_{n}\right)$ and $\left(\phi_{n}\right)$ are strictly decreasing for $n \geq 2$ and all natural numbers $n$, respectively.

Proof. We set $\theta_{n}-\theta_{n+1}=f(n)$, where

$$
\begin{aligned}
f(x) & =-\frac{1}{x+1}-\log \left(x+\frac{1}{2}+\frac{1}{24 x}-\frac{1}{48 x^{2}}+\frac{23}{5760 x^{3}}+\frac{17}{3840 x^{4}}-\frac{10099}{2903040 x^{5}}\right) \\
& +\log \left(x+\frac{3}{2}+\frac{1}{24(x+1)}-\frac{1}{48(x+1)^{2}}+\frac{23}{5760(x+1)^{3}}+\frac{17}{3840(x+1)^{4}}-\frac{10099}{2903040(x+1)^{5}}\right) .
\end{aligned}
$$

Differentiation gives

$$
f^{\prime}(x)=\frac{p(x)}{q(x)}
$$

where

$$
\begin{aligned}
& p(x)=-223661795575-1556403370554 x-4175585115408 x^{2} \\
& -4951284518880 x^{3}-1613300443776 x^{4}+1495234411776 x^{5} \\
& +1016470425600 x^{6}
\end{aligned}
$$

and 


$$
\begin{aligned}
& q(x)=-44732359115 x-285157435534 x^{2}-655259062139 x^{3} \\
& -595777525560 x^{4}-16441205760 x^{5}+7510856117184 x^{6} \\
& +67212592098624 x^{7}+276219358571520 x^{8}+661238937354240 x^{9} \\
& +1014009112166400 x^{10}+1032643563356160 x^{11} \\
& +698089616179200 x^{12}+301990477824000 x^{13} \\
& +75848771174400 x^{14}+8427641241600 x^{15}
\end{aligned}
$$

By expanding $p(x)$ and $q(x)$ as a power series of $x-2$ we get

$$
\begin{aligned}
& p(x)=27439716165461+185481302397702(x-2) \\
& +290969152206768(x-2)^{2}+204586956497952(x-2)^{3} \\
& +74327269209984(x-2)^{4}+13692879518976(x-2)^{5} \\
& +1016470425600(x-2)^{6},
\end{aligned}
$$

and

$$
\begin{aligned}
& q(x)=10423677493515991770+62668051134141291321(x-2) \\
& +\ldots+328678008422400(x-2)^{14}+8427641241600(x-2)^{15},
\end{aligned}
$$

which is a polynomial with all positive coefficients. It follows $f^{\prime}(x)>0$ for $x \geq 2$, so that $f$ is strictly increasing in $(2, \infty)$ with $\lim _{x \rightarrow \infty} f(x)=0$. it results that $f(x)<0$ for $x \geq 2$, namely $\theta_{n}$ is strictly increasing for $n \geq 2$. This completes the first part of Theorem 2.3. To prove the second part of the theorem we denote $\phi_{n}-\phi_{n+1}=g(n)$, where

$$
\begin{aligned}
& g(x)=-\frac{1}{x+1}-\log \left(x+\frac{1}{2}+\frac{1}{24\left(x+\frac{1}{2}\right)}-\frac{37}{5760\left(x+\frac{1}{2}\right)^{3}}\right. \\
& \left.+\frac{10313}{2903040\left(x+\frac{1}{2}\right)^{5}}\right)+\log \left(x+\frac{3}{2}+\frac{1}{24\left(x+\frac{3}{2}\right)}-\frac{37}{5760\left(x+\frac{3}{2}\right)^{3}}+\frac{10313}{2903040\left(x+\frac{3}{2}\right)^{5}}\right) .
\end{aligned}
$$

By differentiation we get

$$
g^{\prime}(x)=\frac{t(x)}{s(x)}
$$

where

$$
\begin{aligned}
& t(x)=9678358492223+57880272188784 x+144357200961720 x^{2} \\
& +192184418280960 x^{3}+144005296337280 x^{4}+57575515060224 x^{5} \\
& +9595919176704 x^{6}
\end{aligned}
$$

and

$$
\begin{aligned}
& s(x)=5912418259515+110278703811038 x+996749749920191 x^{2} \\
& +\ldots+539369039462400 x^{15}+33710564966400 x^{16},
\end{aligned}
$$

which is a polynomial with all positive coefficients. Since both $t(x)$ and $s(x)$ are positive for $x \geq 1, g$ is strictly increasing with $\lim _{x \rightarrow \infty} g(x)=0$, consequently, the sequence $\left(\phi_{n}\right)$ is strictly increasing for $n=1,2,3, \ldots$. This completes the proof of Theorem 2.3. 
As a direct consequence of the fact that $\theta$ is strictly increasing for $n=2,3,4, \ldots$ we have $\theta_{2} \leq \theta_{n}<\lim _{n \rightarrow \infty} \theta_{n}=\gamma$ for all $n \geq 2$. As $\theta_{2}=\frac{3}{2}-\log \left(\frac{58804553}{23224320}\right)$, we have

Corollary 2.4. Let $n \geq 2$ be an integer. Then we have

$$
\begin{aligned}
& \alpha+\log \left(n+\frac{1}{2}+\frac{1}{24 n}-\frac{1}{48 n^{2}}+\frac{23}{5760 n^{3}}+\frac{17}{3840 n^{4}}-\frac{10099}{2903040 n^{5}}\right) \\
& \leq H_{n}<\beta+\log \left(n+\frac{1}{2}+\frac{1}{24 n}-\frac{1}{48 n^{2}}\right. \\
& \left.+\frac{23}{5760 n^{3}}+\frac{17}{3840 n^{4}}-\frac{10099}{2903040 n^{5}}\right),
\end{aligned}
$$

where $\alpha=\frac{3}{2}-\log \left(\frac{58804553}{23224320}\right)=0.5709807216 \ldots$ and $\beta=\gamma=0.5772156 \ldots$ are the best possible.

Similarly from monotonic increase of the sequence $\left(\phi_{n}\right)$ with $\lim _{n \rightarrow \infty} \phi_{n}=$ $\gamma$ and $\phi_{1}=1-\log \left(\frac{6729631}{4408992}\right)=0.57712577887 \ldots$, we get

$$
\begin{gathered}
\alpha^{*}+\log \left(n+\frac{1}{2}+\frac{1}{24\left(n+\frac{1}{2}\right)}-\frac{37}{5760\left(n+\frac{1}{2}\right)^{3}}+\frac{10313}{2903040\left(n+\frac{1}{2}\right)^{5}}\right) \leq H_{n} \\
\beta^{*}+\log \left(n+\frac{1}{2}+\frac{1}{24\left(n+\frac{1}{2}\right)}-\frac{37}{5760\left(n+\frac{1}{2}\right)^{3}}+\frac{10313}{2903040\left(n+\frac{1}{2}\right)^{5}}\right),
\end{gathered}
$$

where $\alpha^{*}=1-\log \left(\frac{6729631}{4408992}\right)=0.57712577887 \ldots$ and $\beta^{*}=\gamma=0.5772156$ are the best possible constants.

\section{References}

[1] N. Batir, Sharp bounds for the psi function and harmonic numbers, Math. Inequal. Appl., No. 4, pp. 917-925, (2011).

[2] C-P Chen, C. Mortici, New sequences converging towards the EulerMascheroni constant, Computer and Mathematics with Applications, doi:10.1016/j.camwa.2011.03.099, (2011).

[3] C-P. Chen, Inequalities for the Euler-Mascheroni constant, Appl. Math. Lett., 23, pp. 161-164, (2010). 
[4] C. Mortici, New approximation of the gamma function in terms of the digamma function, Appl. Math. Lett., 23, No. 1, pp. 97-100, (2010).

[5] C. Mortici, Fast convergences toward Euler-Mascheroni constant, Comput. Appl. Math., 29, No. 3, pp. 479-491, (2010).

[6] C. Mortici, On new sequences converging towards the Euler-Mascheroni constant, Computer Math. Appl., 59, No. 8, pp. 2610-2614, (2010).

[7] C. Mortici, Optimizing the rate of convergence of some new classes of sequences convergent to Euler constant, Analysis Appl., 8, No. 1, pp. 99-107, (2010).

[8] C. Mortici, A quicker convergence toward the constant with the logarithm term involving the constant e, Carpathian J. Math., 26, No. 1, pp. 86-91, (2010).

[9] T. Negoi, A faster convergence to the constant of Euler, Gazeta Matematica, Seria A, 15, No. 94, pp. 113, (1997).

[10] D. W. Temple, A geometric look at sequences that converge to Euler's constant, College Math. J., 37, pp. 128-131, (2006).

[11] D. W. Temple, A quicker convergences to Euler's constant, Amer. Math. Monthly, 100 (5), pp. 468-470,(1993).

[12] R. M. Young, Euler's constant, Math. Gaz., 75, pp. 187-190, (1991).

\section{Necdet Batir}

Department of Mathematics, Faculty of Arts and Sciences, Nevşehır University, Nevşehır, Turkey

e-mail : nbatir@hotmail.com

and

\section{Chao-Ping Chen}

School of Mathematics and Informatics, Henan Polytechnic University, Jiaozuo City 454003, Henan Province, People's Republic of China e-mail : chenchaoping@sohu.com 